\title{
DESIGN, MANUFACTURING AND TESTING OF NOVEL HIGH-RESOLUTION OPTICAL PAYLOAD FOR REMOTE SENSING SATELLITE
}

\author{
Spencer Lynch ${ }^{1}$, Rachel Dunn ${ }^{1}$, Daniel Wartman ${ }^{1}$, Patrick Walsh ${ }^{1}$, Anne-Sophie Poulin-Girard ${ }^{3}$, Simon Thibault ${ }^{3}$, \\ Grant McSorley ${ }^{1}$, William Whelan ${ }^{2}$, Nicholas Krouglicof ${ }^{1}$ \\ ${ }^{1}$ Faculty of Sustainable Design Engineering, University of Prince Edward Island, Charlottetown PE, Canada \\ ${ }^{2}$ Department of Physics, University of Prince Edward Island \\ ${ }^{3}$ Laboratoire de Recherche en Ingénierie Optique, Université de Laval
}

\begin{abstract}
Through the Canadian CubeSat Project (funded by the Canadian Space Agency), the Faculty of Sustainable Design Engineering (FSDE) is designing and building a remote sensing CubeSat, called SpudNik-1. The CubeSat will use high resolution satellite imagery (HRSI) to capture regions of agriculture in the province. This image data will be relayed to a ground station constructed at the FSDE, where image data will be used to study crop health, weed infestation and other metrics for precision agriculture. This information will be shared with local farmers to assist with increasing crop yields through precise pesticide and nutrient application.

Using a reflective-based Cassegrain telescope design fitted to the $2 \mathrm{U}$ CubeSat size constraints, the imaging payload onboard SpudNik-1 will capture HRSI with a $2-10 \mathrm{~m}$ ground sampling distance. Images will be captured in the visible spectrum, from 380 to $750 \mathrm{~nm}$, in low Earth orbit using a 5.3 MP CMOS sensor (Python 5000, ON Semiconductor) fitted with a global shutter. The mass of the imaging subsystem has a weight target of 650 grams and an effective focal length (EFL) of $480 \mathrm{~mm}$. By folding the EFL using a pair of $45^{\circ}$ mirrors, the payload occupies $160 \mathrm{~mm}$ of the total $227 \mathrm{~mm}$ available in the $2 \mathrm{U}$ CubeSat platform along the Z-axis.

Through a partnership with the UPEI Physics Department and Université Laval's Laboratoire de Recherche en Ingénierie Optique (LRIO), optical analyses were performed using Zemax OpticStudio. Through this, ray tracings, optical design tolerances and the EFL have been simulated. Additionally, the prototype mirrors for the FlatSat model were manufactured at LRIO. Following alignment, radiometric and geometric resolution will be determined to assess overall image quality with the prototype mirrors.

The payload will be verified to meet design requirements through three key testing stages. These stages include preliminary alignment according to the Zemax simulation to confirm the payload geometry, a payload prototype integrated with the FlatSat, and the flight model. The FlatSat payload prototype will include fixed optics, matte black anodized aluminum, low outgassing epoxy and O-rings suited for aerospace applications, which will be analogous to the flight model. The FlatSat will integrate the optical payload and on-board computer prototype, allowing images to be captured and tested using two critical subsystems.
\end{abstract}

This presentation will include design details of the optical system up to its current stage, integration of the payload into the CubeSat, and student and faculty perspectives on the project. 